\title{
Photothermal treatment of glioma; an in vitro study of macrophage-mediated delivery of gold nanoshells
}

\author{
Seung-Kuk Baek • Amani Riad Makkouk • \\ Tatiana Krasieva $\cdot$ Chung-Ho Sun • \\ Steen J. Madsen • Henry Hirschberg
}

Received: 1 September 2010/Accepted: 20 December 2010/Published online: 9 January 2011

(C) The Author(s) 2011. This article is published with open access at Springerlink.com

\begin{abstract}
One of the major factors that limits the treatment effectiveness for gliomas is the presence of the blood-brain barrier (BBB) which protects infiltrating glioma cells from the effects of anti-cancer agents. Circulating monocytes/macrophages (Ma) have a natural ability to traverse the intact and compromised BBB and loaded with anti cancer agents could be used as vectors to target tumors and surrounding tumor infiltrated tissue. Nanoshells (NS) are composed of a dielectric core (silica) coated with an ultrathin gold layer which converts absorbed near-infrared light (NIR) to heat with an extremely high efficacy and stability. We have investigated the effects of exposure to laser NIR on multicell human glioma spheroids infiltrated with empty (containing no nanoshells) or nanoshell loaded macrophages. Our results demonstrated that; (1) macrophages could efficiently take up bare or coated (PEGylated) gold NS: (2) NS loaded macrophages infiltrated into glioma spheroids to the same or, in some cases, to a greater degree than empty Ma; (3) NIR laser irradiation of spheroids incorporating NS loaded macrophages resulted in complete growth inhibition in an irradiance dependent manner, and (4) spheroids infiltrated with empty macrophages had growth curves identical to untreated control
\end{abstract}

S.-K. Baek · T. Krasieva · C.-H. Sun · S. J. Madsen ·

H. Hirschberg $(\square)$

Beckman Laser Institute, University of California, Irvine, 1002 Health Sciences Rd, Irvine, CA 92617, USA

e-mail: hhirschb@uci.edu

S.-K. Baek

Department of Otolaryngology-Head and Neck Surgery, Korea

University College of Medicine, Seoul, Korea

A. R. Makkouk · S. J. Madsen · H. Hirschberg

Department of Health Physics and Diagnostic Sciences,

University of Nevada, Las Vegas, NV, USA cultures. The results of this study provide proof of concept for the use of macrophages as a delivery vector of NS into gliomas for photothermal ablation and open the possibility of developing such regimens for patient treatment.

Keywords Gold nanoshells - Macrophage . Nanoshell delivery $\cdot$ Blood brain barrier · Gliomas

\section{Introduction}

Malignant gliomas are the most common primary brain tumors in adults. The most aggressive of these tumors, glioblastoma multiforme (GBM), are characterized by a number of histological features including significant proliferation, invasion, angiogenesis and necrosis. Even with substantial improvements in conventional treatments consisting of surgery, radiation therapy and chemotherapy, the prognosis for patients with this disease has not improved significantly over the past four decades: median survival is approximately $12-18$ months. One of the major factors that limits the treatment effectiveness for gliomas is the presence of the blood-brain barrier (BBB) which protects infiltrating glioma cells from the effects of anti-cancer agents [1-3]. Failure to eradicate these infiltrating cells invariably results in tumor recurrence and is responsible for the dismal prognosis of GBM patients.

The BBB is formed by tightly connected brain capillary endothelial cells that prevent passage of most biological molecules. This impermeability prevents harmful substances in the blood plasma from entering the brain. Unfortunately this protective barrier also prevents anticancer agents from reaching infiltrating glioma cells that have migrated from the solid tumor mass. Nearly all largemolecule pharmaceutics (peptides, recombinant proteins, 
monoclonal antibodies and gene therapeutics) and $98 \%$ of small molecules do not cross the BBB $[4,5]$.

Circulating monocytes/macrophages (designated $\mathrm{Ma}$ in this paper) have a natural ability to traverse the intact and compromised BBB and undergo differentiation into longlived brain-resident macrophages and microglia [6]. Tumor-associated Ma are frequently found in and around GBM in both experimental animals and patient biopsies [7-10]. Additionally, intravenously injected Ma loaded with iron oxide nanoparticles have been shown to accumulate in and at the border of experimental brain tumors [11]. These observations indicate local synthesis of chemo attractive factors in gliomas and that inflammatory cells can pass through the blood brain tumor barrier (BBTB) as well as the brain adjacent to tumor (BAT) region where the concentration of infiltrating glioma cells is greatest. Ma loaded with drugs, nanoparticles or photosensitizers could therefore be used as vectors to target tumors and surrounding tumor infiltrated tissue [11-15].

Nanoshells are a new class of optically tunable nanoparticle (designated NS) composed of a dielectric core (silica) coated with an ultrathin metallic layer (gold) [1618]. By adjusting the relative core and shell thickness, nanoshells can be manufactured to absorb or scatter light at near-infrared (NIR) wavelengths, a region of light where optical penetration through tissue is optimal. Furthermore, the metal shell converts absorbed NIR to heat with an efficacy and stability that far exceeds that of conventional dyes. Not only do gold-silica nanoshells have advantageous optical properties, but their size allows them to be taken up and accumulated in quantity in Ma.

In the study reported here we have investigated the effects of exposure to NIR on multicell human glioma spheroids infiltrated with empty (containing no NS) or NS loaded Ma. Spheroid cultures were used since they are well suited to studies of the direct effects on tumors of modalities like photodynamic therapy (PDT) or photothermal therapy (PTT) without the effects of circulatory or immunological influences [19]. The results of this study provide proof of concept for the use of macrophages as a delivery vector of NS into gliomas for photothermal ablation and open the possibility of developing such regimens for patient treatment.

\section{Materials and methods}

Cell cultures of brain tumor spheroid and macrophages

The human grade IV glioma cell line (ACBT) and murine Ma P388-D1 (ATCC, CCL-46) were used in all experiments. The cells were cultured in Dulbecco's Modified Eagle Media (DMEM, Gibco, Carlsbad, CA) with high glucose and supplemented with $2 \mathrm{mM}$ L-glutamine, gentamycin $(100 \mathrm{mg} / \mathrm{ml})$, and $2 \%$ heat-inactivated fetal bovine serum (Gibco). Cells were maintained at $37^{\circ} \mathrm{C}$ in a $7.5 \%$ $\mathrm{CO}_{2}$ incubator.

\section{Nanoshells}

The gold nanoshells (NS) used in this study consisted of a $120 \mathrm{~nm}$ silica core with a $12-15 \mathrm{~nm}$ gold shell (Nanospectra Biosciences, Inc., Houston, Texas). The resultant optical absorption peak was between 790 and $820 \mathrm{~nm}$ for both bare and PEGylated particles. The absorbance curves of bare (a) and PEGylated (b) nanoshell solutions as supplied by the manufacturer are shown in Fig. 1. The solutions were found to have an optical density (O.D.) of 1.6 at $\lambda=819 \mathrm{~nm}$ for bare nanoshells (no dilution) and 1.22 at $\lambda=795 \mathrm{~nm}$ for PEGylated nanoshells $(100 \times$ dilution).

The uptake of nanoshells in murine macrophages was studied using a UV-Vis-NIR spectrophotometer. $5 \times 10^{6}$ P388-D1 cells were incubated with either $4.3 \times 10^{9}$ bare nanoshells or $1.4 \times 10^{11}$ PEGylated nanoshells $(1 \mathrm{ml})$ for $24 \mathrm{~h}$ at $37^{\circ} \mathrm{C}$ in $5 \% \mathrm{CO}_{2}$. Following incubation, macrophages were transferred to $15-\mathrm{ml}$ centrifuge tubes, centrifuged at $600 \mathrm{rpm}$ for $7 \mathrm{~min}$, and then washed twice with PBS to remove excess particles. The final cell pellet was suspended in $3 \mathrm{ml}$ of DMEM, and the absorbance of the resultant cellular suspension measured with a Varian UV-Vis-NIR spectrophotometer (Cary 6000i, Varian, USA). Absorbance was measured between 600 and $1100 \mathrm{~nm}$, which covers the broad absorption peak of both bare and PEGylated nanoshells. To calculate \% uptake of NS, reference solutions containing NS in the absence of Ma were used, and were prepared in the same media as that used for macrophage incubation, and at the same dilution ratio. Background correction was necessary to

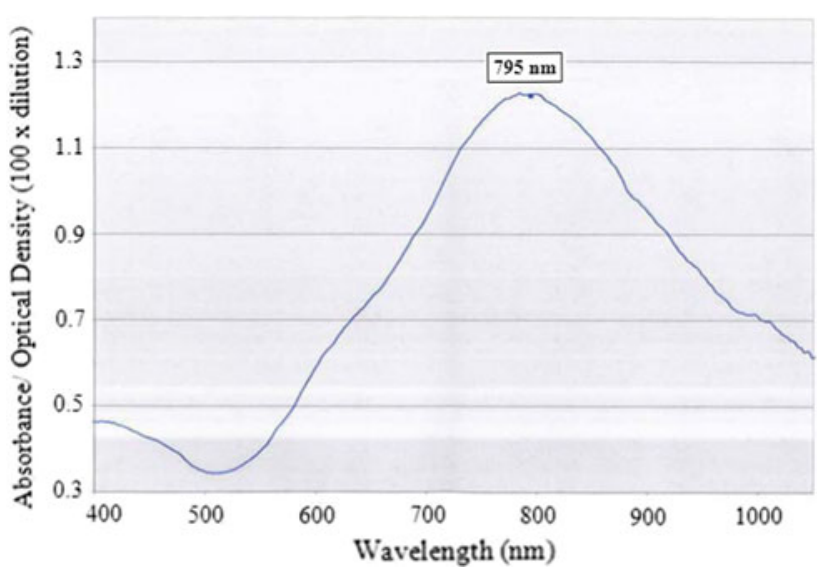

Fig. 1 The absorbance curves of PEGylated nanoshell solutions as supplied by the manufacturer. The solution was found to have an optical density (O.D.) 1.22 at $\lambda=795 \mathrm{~nm}(100 \times$ dilution) 
obtain absorbance values corresponding to NS only, i.e. without medium interference. Consequently, solutions prepared by substituting the NS volume added with deionized water were measured, and their absorbance values subtracted from the NS solution absorbance values to give the pure absorbance of the NS. The absorbance of NS only, i.e. without macrophage interference is needed to calculate \% uptake. Consequently, macrophages incubated under similar conditions (same number, total volume, incubation time) in absence of NS were similarly washed and suspended in $3 \mathrm{ml}$ DMEM. The resulting solution was measured, and its absorbance value subtracted from those of the macrophage groups to give the pure absorbance of the incorporated nanoparticles. \% uptake of nanoshells was calculated by the absorbance of incorporated nanoshells at $\lambda=819 \mathrm{~nm}$ divided by the absoption of the nanoshells alone $\times 100$.

Generation of tumor cell and hybrid tumor/Ma spheroids

Spheroids were formed by a modification of the centrifugation method first described by Ivascu and Kubbies [20]. Tumor spheroids were generated with $5 \times 10^{3}$ cells in $200 \mu \mathrm{l}$ of culture medium per well of an ultra-low attachment surface 96-well round bottomed plate (Corning Inc., NY). To form hybrid spheroids, Ma were mixed with tumor cells at ratios of tumor cells:Ma ranging from 2:1 to $10: 1$ (50-10\%). In all cases, $5 \times 10^{3} /$ well of tumor cells were used. Empty and NS loaded macrophages were treated, prior to their addition to the tumor cells, with mitomycin $\mathrm{C}$ to prevent cell division and their contribution to spheroid growth. The plates were centrifuged at $1000 \times \mathrm{g}$ for $10 \mathrm{~min}$. Immediately following centrifugation the tumor cells formed into a disk shape. The plates were maintained at $37^{\circ} \mathrm{C}$ in a $7.5 \% \mathrm{CO}_{2}$ incubator for $48 \mathrm{~h}$ to allow them to take on the usual 3 dimensional spheroid form.

\section{Fluorescence labeling of Ma}

$20 \times 10^{6} \mathrm{Ma} / \mathrm{ml}$ were labeled with fluorescence cell tracking dye PKH26GLred (SigmaAldrich, St. Louis, MO). After washing twice in basal medium without serum, cell pellets were resuspended in $1 \mathrm{ml}$ Diluent $\mathrm{C}$ (an iso-osomotic aqueous solution without physiologic salts or buffers) by gentle pipetting. The cell suspension in Diluent $\mathrm{C}$ was rapidly added to $1 \mathrm{ml}$ of $2 \times$ dye concentration at $4 \times 10^{-6}$ molar by gentle pipetting. After $5 \mathrm{~min}$ of incubation at room temperature, an equal amount of FBS was added to stop the staining process. Additional three washes with $10 \mathrm{ml}$ complete growth medium each to remove any residue unbounded dyes were carried out to complete the labeling process.
Uptake of nanoshells in labeled Ma

Labeled Ma were seeded in $35 \mathrm{~mm}$ cell culture dishes at $10^{6}$ $\mathrm{Ma}$ in $2 \mathrm{ml}$ of the culture medium. The dishes were incubated overnight to allow the cells to settle and adhere to the plastic. Culture medium was exchanged for $100 \mu$ lof either bare $\left(3.2 \times 10^{9}\right.$ particles $\left./ \mathrm{ml}\right)$ or PEGylated $\left(2.8 \times 10^{11}\right.$ particles/ml) nanoshells colloid in $1.9 \mathrm{ml}$ of culture medium. The Ma were incubated for $24 \mathrm{~h}$ at $37^{\circ} \mathrm{C}$, rinsed three times with Hanks' Balanced Salt Solution with calcium chloride and magnesium chloride (HBSS, Gibco, Carlsbad, CA) to wash away the excess of non-ingested nanoshells. Nanoshell laden Ma were then detached with trypsin, washed and counted.

\section{Photothermal treatment (PTT)}

Individual spheroids in each well of a 96 well round bottomed plate were irradiated with $810 \mathrm{~nm}$ laser light (Coherent Inc., Santa Clara, CA) at irradiances ranging from 2 to $28 \mathrm{~W} / \mathrm{cm}^{2}$ with a laser spot size approximately 3 or $5 \mathrm{~mm}$ diameter. Laser treatment lasted for exposure intervals of 1,5 or $10 \mathrm{~min}$. At various time intervals ranging from 1 to 14 days following PTT, the cells were stained using a combination of Hoechst 33342 and Ethidium Homodimer 1 (Invitrogen H1399, Carlsbad, CA) and were visualized using an inverted Zeiss laser-scanning microscope (LSM 410, Carl Zeiss, Jena, Germany). This system allows the differential visualization of cell nuclei using confocal and two-photon microscopy. Simultaneously detected blue and red emissions were isolated by using BP 390-465 IR and BP 565-615 IR band pass filters, respectively. Fluorescent images were pseudo-colored blue and red.

Statistical analysis

The effect of PTT on the growth of spheroids containing; 1. No Ma, 2. empty Ma or 3. NS loaded Ma was compared using the Student's $t$-test for unrelated samples. A bidirectional hypothesis was applied and significance was considered when the $P$-value was less than 0.05 .

\section{Results}

Macrophage endocytosis of bare or PEGylated nanoshells

Bright Field TEM images of murine macrophages incubated for $24 \mathrm{~h}$ with nanoshells revealed extensive intracellular presence of the nanoparticles (Fig. 2). Nanoshells, in small aggregates, were inside vacuoles which were dispersed throughout the cellular cytoplasm (white arrows). 
Fig. 2 TEM images of nanoshell-loaded murine macrophages. The nanoshells are aggregated in vacuoles dispersed throughout the cytoplasm (a and $\mathbf{b}$ ).

Magnification of a vacuole in a show the high contrast nanoshells. White arrows in b indicate vacuoles containing NS
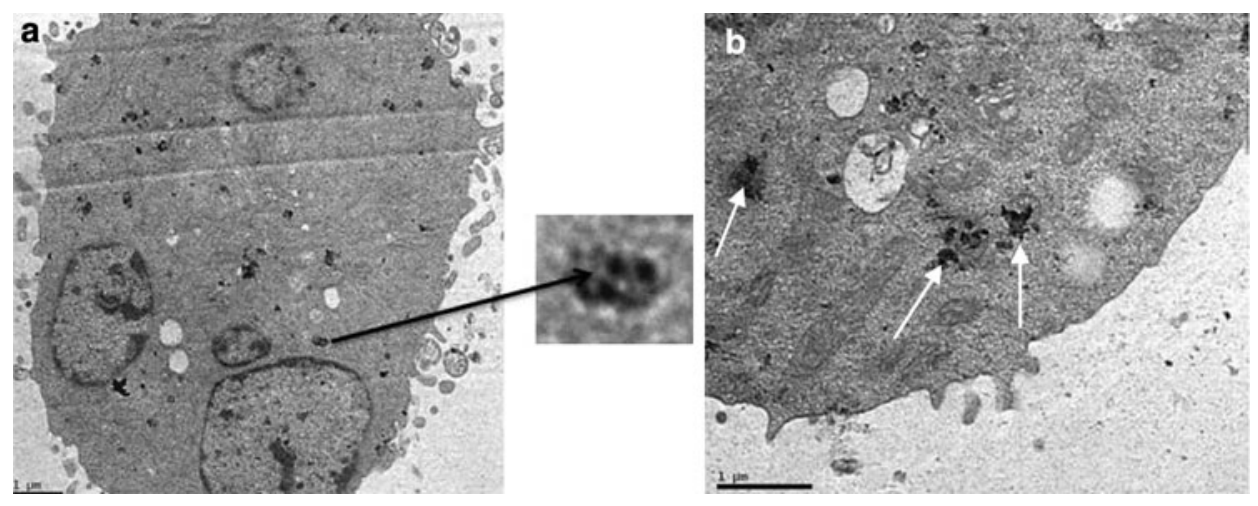

Aggregates of nanoshells in the cytoplasm of macrophages could also be visualized with 2 photon microscopy as shown in Fig. 3.

The \% uptake of PEGylated nanoshells in murine macrophages was lower (3.96\%) than that of bare nanoshells (15.74\%). However, the PEGylated nanoshell solution was available in a much higher concentration since they have a much lower tendency to aggregate compared to bare nanoshells. Comparison was therefore between the respective numbers of nanoshells taken up by a given number of macrophages ( 5 million in both groups). Ma incorporated $59 \times 10^{8}$ PEGylated nanoshells versus only $6.8 \times 10^{8}$ bare nanoshells. In other words, the total amount of PEGylated nanoshells taken up by the macrophages was more than 8 times that of bare nanoshells. PEGylated nanoshells were therefore used in all subsequent experiments.

\section{Generation of hybrid spheroids}

Hybrid spheroids were produced by the centrifugation method containing varying tumor to macrophage cell ratios ranging from a $2: 1$ to a $10: 1$ ratio. A typical hybrid spheroid produced in this manner with a tumor cell:Ma ratio of 2:1 is shown in Fig. 4a. The macrophages were labeled with PKH26GLRed to allow their identification with two photon fluorescent microscopy within the spheroid. As can be seen from the figure the macrophages were more or less equally distributed throughout the spheroid at this ratio. No Ma were observed outside the spheroid indicating that all were incorporated during centrifugation. By careful control of the number of Ma mixed with the tumor cells (ratio TC:MA 10:1) and the centrifugation RPM, it proved possible to generate spheroids where the Ma accumulated in the core of the spheroid.

Migration of empty or NS-loaded macrophages into preformed spheroids

ACBT human tumor spheroids were generated by the centrifugation method as previously described. Forty-eight hours following their generation, $2 \times 10^{4} / \mathrm{ml}$ PKH26GL Red labeled empty or nanoshell loaded Ma were added to the wells and co incubated for 4, 18 and $36 \mathrm{~h}$. The number of labeled Ma that had infiltrated into the spheroids increased for the first $12 \mathrm{~h}$ of co-incubation and then appeared to approach saturation levels. The distribution of infiltrating labeled Ma (Fig. 4b, c) assayed after $12 \mathrm{~h}$ of coincubation was similar to that seen in the hybrid spheroids (Fig. 4a). Loaded macrophages appeared to infiltrate at an increased initial rate and penetrated deeper into the spheroid during the first $2 \mathrm{~h}$ of co-incubation. The majority of empty Ma were found in the outer rim of the spheroid at the $2 \mathrm{~h}$ time point, while in contrast, NS loaded Ma had a much deeper penetration during this initial incubation period. By the $12 \mathrm{~h}$ time point, no significant differences between empty and loaded Ma could be determined when comparing either number of Ma or their penetration depth (data not shown).

Effects of PTT on hybrid and infiltrated spheroids

Hybrid or infiltrated spheroids containing either empty or NS loaded Ma were exposed to NIR irradiation of increasing irradiances. Spheroid cultures were stained with the live/dead markers Hoechst 33342 and Ethidium Homodimer $24 \mathrm{~h}$ before evaluation by laser scanning microscopy. As can be seen from Fig. 5 there was a striking difference in spheroid survival between those containing empty macrophages compared to those containing NS loaded macrophages. Laser treatment not only caused the destruction of the loaded Ma but also was toxic to the surrounding tumor cells (Fig. 5c, d). In contrast, spheroids containing empty Ma showed no significant increase in necrotic cells following laser irradiation compared to nontreated controls (Fig. 5 a and b).

PTT effect on hybrid spheroid growth

Hybrid spheroids were allowed to grow in culture for a period of 12 days to determine their viability following 
Fig. 3 Two photon fluorescence micrograph of a empty or b nanoshell loaded Ma. Cell nucleus stained with Hoechst 33342 (blue),

Cytoplasm stained with PKH26 Red Fluorescent (red), nanoshell aggregates inside $\mathrm{Ma}$ shown by white reflectance (white arrows)
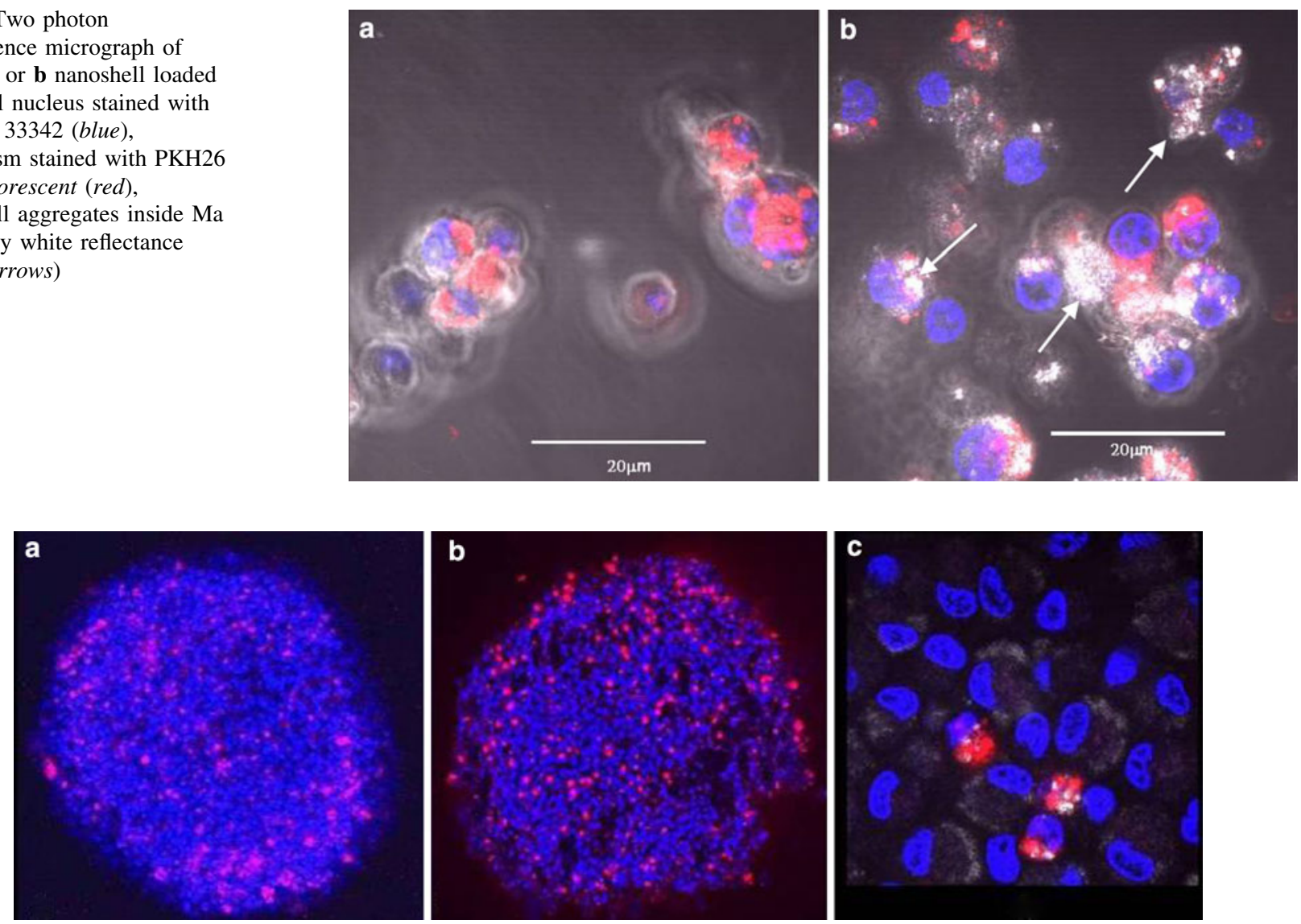

Fig. 4 Two photon fluorescence image of a hybrid (tumor cell/Ma) spheroid, b Ma infiltrated spheroid. c nanoshell loaded macrophage migration into tumor spheroids. Cell nucleus stained with Hoechst 33342 (blue), Ma cytoplasm stained with PKH26 Red Fluorescent

PTT. PTT was administered at an irradiance of $14 \mathrm{~W} / \mathrm{cm}^{2}$ for $10 \mathrm{~min}$. The kinetics of the hybrid spheroid growth pattern is shown in Fig. 6A. Hybrid spheroids containing empty Ma (b) displayed no significant decrease in their growth pattern following PTT compared to that observed for spheroids containing either empty (a) or NS loaded (c) Ma receiving no radiation $(P=0.074)$. In contrast PTT had a highly significant effect on NS loaded Ma spheroid survival $(d, e)$ with a complete cessation of growth in all of the spheroids examined $(P<0.001)$. There were no significant differences in the effects of PTT on spheroids containing either $50 \%$ (d) or $20 \%$ (e) Ma of the total number of cells $(P=0.29)$, both showing a significant reduction in size from day 1 to day 12 after laser treatment. Since the spheroids were generated with $5 \times 10^{3}$ tumor cells, $1 \times 10^{3}$ loaded Ma were sufficient to cause none of the spheroids to survive PTT. This was in contrast to non laser treated spheroids which demonstrated rapid growth, increasing their average diameter from 700 to $1100 \mu \mathrm{m}$, corresponding to an increase in volume of 6-7 times. (red), nanoshell aggregates inside Ma shown by white reflectance. Images were acquired at a depth of approximately $80 \mu \mathrm{m}$. The field of view for images $600 \times 600 \mu \mathrm{m}(\mathbf{a}, \mathbf{b}) 40 \times 40 \mu \mathrm{m}(\mathbf{c})$

\section{PTT effect on Ma infiltrated formed spheroids}

The effects of PTT on spheroids infiltrated in co-culture with empty or NS loaded Ma was also evaluated (Fig. 6B).

Spheroids were formed containing $5 \times 10^{3}$ tumor cells. $48 \mathrm{~h}$ after formation, individual spheroids were co-incubated with $2 \times 10^{4}$ Ma for 2 or $18 \mathrm{~h}$ in the wells of round bottomed 96 well plates. The spheroids were washed and transferred to new wells to remove non-incorporated NS loaded Ma. Following either 2 or $18 \mathrm{~h}$ of co-incubation, sufficient numbers of NS loaded Ma infiltrated into the spheroid to completely inhibit their growth following PTT (Fig. 6B c, d) compared to non treated controls or laser treated controls containing empty $\mathrm{Ma}(P<0.001$; Fig. 6B, $a, b)$.

The effects of increasing laser irradiance are demonstrated in Fig. 7. On day 2 following spheroid formation, $2 \times 10^{4}$ empty or NS loaded Ma were added to each well for an additional $24 \mathrm{~h}$. The spheroids were carefully washed and transferred to new plates and irradiated with either a $5 \mathrm{~mm}\left(2\right.$ and $\left.7 \mathrm{~W} / \mathrm{cm}^{2}\right)$ or a $3 \mathrm{~mm}$ laser spot size 

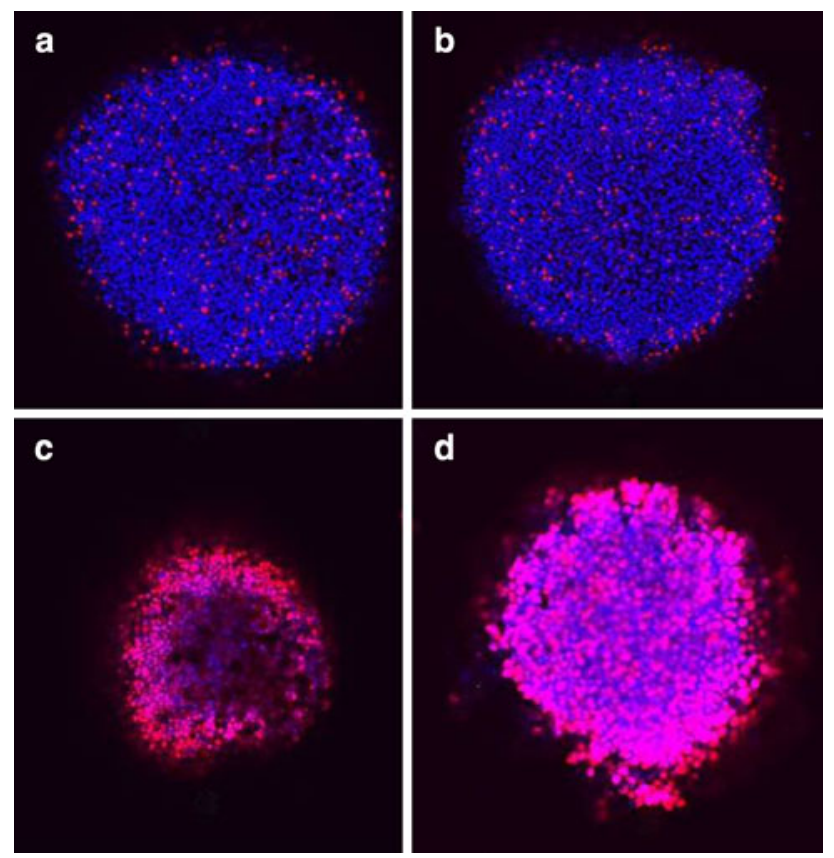

Fig. 5 Live/dead assay of PTT treated hybrid and infiltrated spheroids. Two photon micrographs were made at planes $100 \mu \mathrm{m}$ into the spheroids with simultaneous excitation of the two dyes (Blue live, red dead) a Tumor $+\mathrm{Ma} / \mathrm{NS}$ control. b Tumor $+\mathrm{Ma}+\mathrm{PTT}$, c Tumor $+\mathrm{Ma} / \mathrm{NS}+\mathrm{PTT}, \mathbf{d}$ Tumor $+\mathrm{Ma} / \mathrm{NS}+\mathrm{PTT}$. Images a, $\mathbf{b}$, c show hybrid spheroids $\left(5 \times 10^{3}\right.$ tumor cells $\left.+2.5 \times 10^{3} \mathrm{Ma}\right)$. Image d shows a Ma/NS infiltrated spheroid (24 h co-incubation). PTT: $14 \mathrm{~W} /$ $\mathrm{cm}^{2}, 10$ min. Images in $\mathbf{a}-\mathbf{c}$ taken 12 days post PTT, image in $\mathbf{d}$ taken 2 days post PTT. Images were acquired at a depth of approximately $60-80 \mu \mathrm{m}$. The field of view for images $600 \times 600 \mu \mathrm{m}$

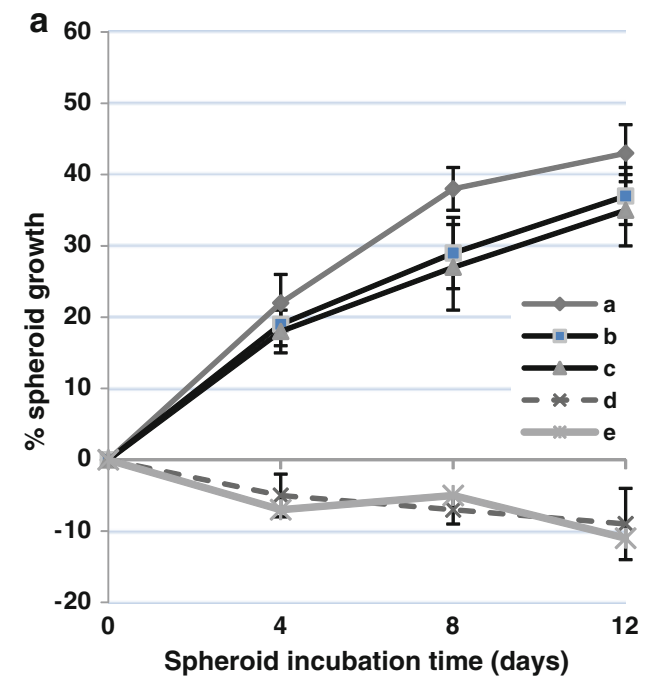

Fig. 6 Kinetics of spheroid growth as a \% initial size following PTT. A Hybrid spheroids were formed containing $5 \times 10^{3}$ tumor cells with $2.5 \times 10^{3}$ empty Ma (a, b), loaded Ma/NS (d, c), or $1 \times 10^{3}$ loaded $\mathrm{Ma} / \mathrm{NS}$ (e) PTT $14 \mathrm{~W} / \mathrm{cm}^{2}, 10 \mathrm{~min}(\mathbf{b}, \mathbf{d}, \mathbf{e})$. B Spheroids were formed containing $5 \times 10^{3}$ tumor cells. $48 \mathrm{~h}$ after formation, for the higher powers and incubated for an additional 14 days. At laser irradiances of $7 \mathrm{~W} / \mathrm{cm}^{2}$, significant inhibition of spheroid growth was observed $(P=0.0023)$ compared to non treated controls. At laser power levels of 14 or $28 \mathrm{~W} / \mathrm{cm}^{2}$ complete suppression of spheroid growth was observed $(P<0.001)$ At the highest power density $\left(28 \mathrm{~W} / \mathrm{cm}^{2}\right)$ employed, spheroids were seen to explode after only seconds of NIR irradiation. In contrast, at all of the power densities used, no significant growth inhibition or delay was seen for spheroids containing either empty $\mathrm{Ma}$ or tumor cells alone compared to spheroids receiving no treatment $(P=0.47)$. Similar results to those observed with a 10 min laser exposure (Fig. 7) were also obtained with an exposure of 5 min (data not shown).

Hybrid spheroids were generated with $5 \times 10^{2}$ loaded Ma concentrated in the core of the spheroid. NIR irradiation at low power for $1 \mathrm{~min}$ had little toxic effect (Fig. 8a) but at an irradiance of $28 \mathrm{~W} / \mathrm{cm}^{2}$ the entire core of the spheroid that contained the loaded Ma was completely obliterated (Fig. 8b, white arrow). Live/dead assay of these spheroids revealed that the surrounding cells in the outer rim, which contained no NS loaded Ma, were affected to a much less dramatic degree (red arrow Fig. 8b).

\section{Discussion}

Tumor resection is usually the first modality employed in the treatment of gliomas. Employing the improved surgical

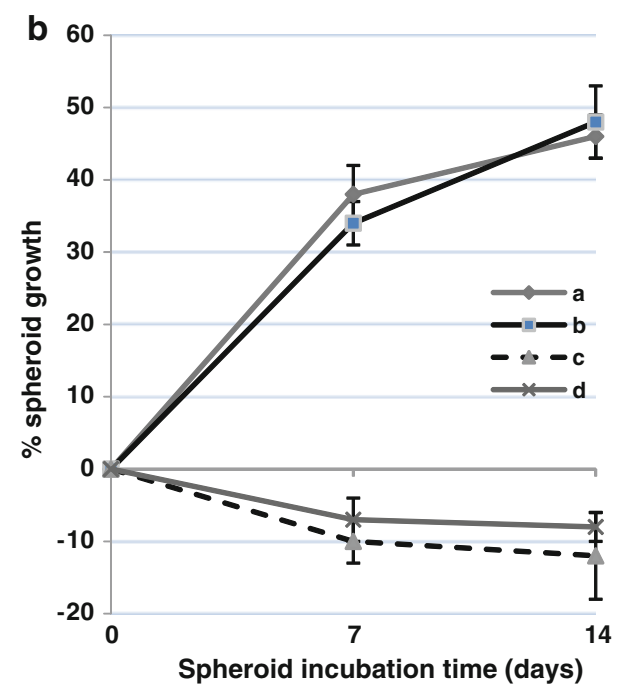

individual spheroids were co-incubated with $2 \times 10^{4}$ empty Ma for $18 \mathrm{~h}$ (b) or NS loaded Ma for $2 \mathrm{~h}$. (d) or $18 \mathrm{~h}$. (a, c) in the wells of round bottomed 96 well plates. The spheroids were washed and transferred to new wells to remove non-incorporated NS. PTT, $14 \mathrm{~W} /$ $\mathrm{cm}^{2}, 10 \min (\mathbf{b}, \mathbf{c}, \mathbf{d})$ 


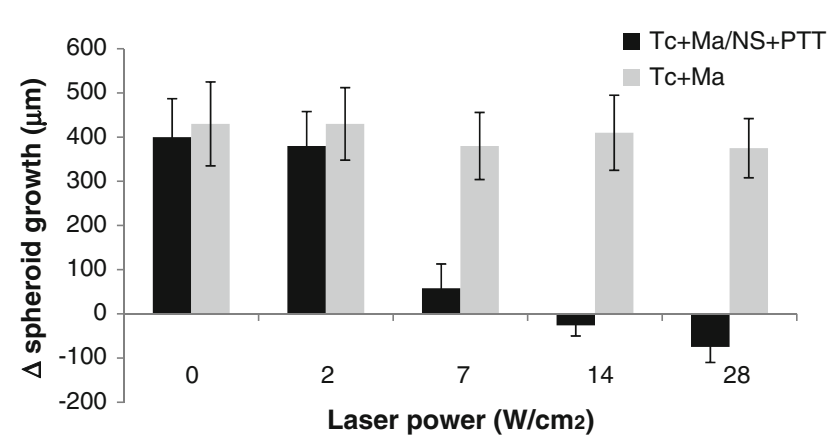

Fig. 7 Effects of laser power on spheroid growth. Preformed spheroids incubated with either empty $(\mathrm{Tc}+\mathrm{Ma})$ or NS loaded $(\mathrm{Tc}+\mathrm{Ma} / \mathrm{NS}) \mathrm{Ma}$ for $24 \mathrm{~h}$, transferred and irradiated with increasing irradiance for $10 \mathrm{~min}$. The values shown represent the average diameter following 14 days of incubation minus the initial diameter of the spheroid ( $\Delta$ spheroid growth)

techniques now available, the incidence of gross tumor resection, as defined by a negative post operative MRI, has greatly increased [21-23]. Nevertheless, the great majority of glioma patients do suffer a recurrence of their tumors leading to their poor prognosis. The therapeutic goal following surgical resection therefore is the elimination of infiltrating tumor cells remaining in the margins of the resection cavity, where most tumor recurrences occur, while minimizing damage to normal brain. One of the many obstacles to effective treatment of malignant brain tumors is limited transport of anti-tumor agents through both brain and brain tumor capillaries due to the bloodbrain barrier $(\mathrm{BBB})$ and the blood-brain tumor barrier (BBTB), the latter which retains many BBB characteristics. $A$ number of methods have been explored for enhancing drug delivery across the BBB/BBTB [24-26]. The ability of macrophages to migrate and accumulate within, and at the periphery, of brain tumors renders them attractive vehicles for the delivery of anti tumor agents including nanoparticles. Ma trafficking into the CNS is a highly regulated function and is dependent on cell-cell interactions that involve tumor cells, endothelial cells and astrocytes. In addition, the local releases of factors that promote BBB permeability also play a role. The use of macrophages as a cell-based delivery vehicle for nanoparticles stemmed from the observations that the iv administration of paramagnetic nanoparticles for MRI, were ingested by endogenous macrophages that subsequently migrated and accumulated in and around tumors [27-29] This was also the case when exogenous in vitro labeled macrophages were injected iv. [11].

The primary objective of this study was to examine the ability of macrophages to act as vehicles that could deliver a sufficient quantity of gold nanoshells into glioma tumor spheroids for efficient PTT. It was hypothesized that: (1) macrophages can efficiently take up bare or PEGylated gold NS, (2) nanoshell-loaded macrophages could infiltrate glioma spheroids following co-incubation to the same degree as empty Ma, (3) NIR laser irradiation of NS loaded Ma infiltrated spheroids would result in significant growth inhibition while spheroids infiltrated with empty macrophages would be undamaged. We have chosen to use this simple in vitro spheroid model since it eliminates many of the problems encountered with monolayer or animal studies. Multicell spheroids are three-dimensional aggregates of tumor cells that mimic micro-tumors prior to their vascularization. Tumor cells within spheroids show a higher degree of morphological and functional differentiation than cells grown in monolayer culture. They also display growth kinetics, metabolic rates, and resistance to radiotherapy and photodynamic and chemotherapy similar to tumor cells in vivo [for review see 19].

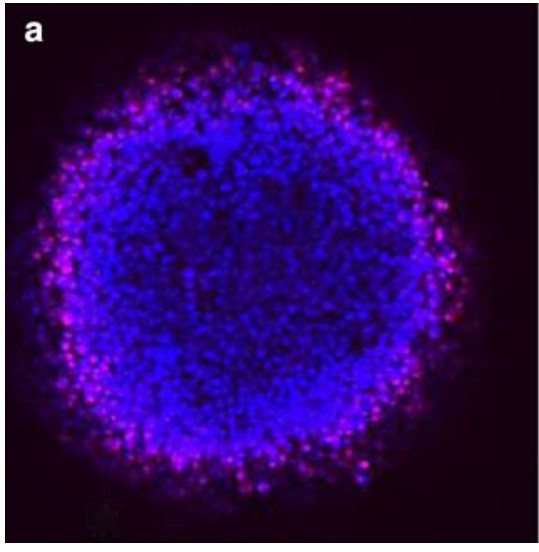

Fig. 8 Two photon micrograph of hybrid spheroid formed with $5 \times 10^{2} \mathrm{Ma} / \mathrm{NS}$ concentrated in the core of the spheroid. a PTT $7 \mathrm{~W} /$ $\mathrm{cm}^{2}$ b PTT $28 \mathrm{~W} / \mathrm{cm}^{2}$ Laser spot size $3 \mathrm{~mm}$, spheroid dia. $0.7 \mathrm{~mm}$. Treatment time $1 \mathrm{~min}$. Images taken $24 \mathrm{~h}$. following treatment. At high irradiance (b) the core of the spheroid was completely

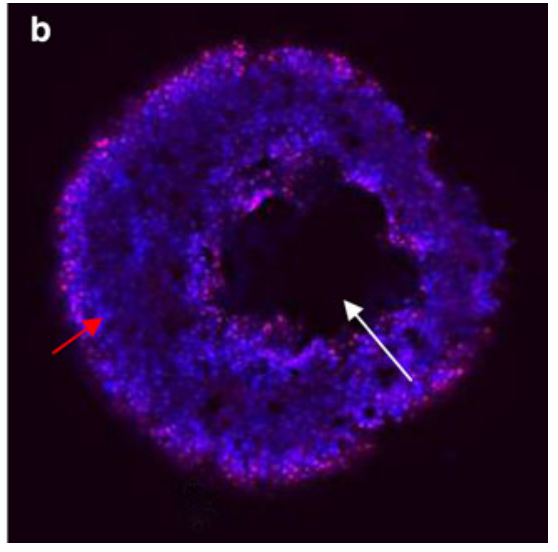

obliterated (white arrow). Live/dead assay of these spheroids revealed that the surrounding cells in the outer rim, which contained no NS loaded $\mathrm{Ma}$, were affected to a much less dramatic degree (red arrow Fig. 8b). Images were acquired at a depth of approximately $80 \mu \mathrm{m}$. The field of view for images $600 \times 600 \mu \mathrm{m}$ 
The ability of Ma to phagocytose several types of nanoparticles including gold nanoshells, the first step in the cell vectorization of NS, has been studied in several previous publications [14, 29]. When nanoparticles are injected into the circulation they are quickly engulfed by fixed (Kupffer cells), spleen, and bone marrow as well as circulating monocytes/macrophages and are rapidly removed from the circulation. Various strategies have been developed in order to prolong their systemic blood circulation time allowing more of these particles to be delivered to the tumor site. Among the various polymers used for this purpose, polyethylene glycol (PEG) is currently the most popular and the most effective in prolonging circulation time of nanoparticles [29, 30] On the other hand, the strategy of employing in vitro loading of macrophage vectors would dictate a maximum uptake of nanoparticles by the macrophages. Although the \% uptake of bare or PEGylated nanoshells was 16 and 4\% respectively, PEGylated nanoshells were available in higher concentration since they have a much lower tendency to aggregate. Therefore the total amount of PEGylated nanoshells taken up by the macrophages was more than 8 times that of bare nanoshells. Additionally, incorporation of nanoshells into the macrophages proved to be nontoxic, as judged with live/dead assays, probably due to the biocompatibility of gold. Spheroids containing empty or NS loaded Ma were produced by two methods. Hybrid spheroids containing mixtures of tumor cells and Ma were centrifuged together in the wells of microplates. The uniform distribution of loaded Ma infiltrating into the spheroids demonstrated in this study is in contrast to previous workers [13, 14] who had shown that macrophages infiltrate the spheroid and accumulate in the inner hypoxic rim around the central necrosis. This also appears to be the case in vivo since tumor associated macrophages (TAMs) have also been seen in high density in hypoxic tumor areas, where it is hypothesized that, being phagocyte scavenger cells, they are attracted by signals released by cells undergoing necrosis. In addition, it has been shown that hypoxic tumor cells recruit TAMs by an increased rate of production of macrophage chemo attractants such as VEGF. Following their migrations, hypoxic tumor cells entrap TAMs by down regulating chemokine receptors and chemo-attractants and up regulating macrophage migration inhibitory factor. This also seems to be the case in GBM but since most or all of the necrotic core often found in gliomas is removed during cytoreductive surgery, it is the TAMs, that have infiltrated the BAT region, which are of therapeutic interest. The even distribution of infiltrating Ma found in our results, in contrast to previous work, can be explained by the observation that spheroids formed by the centrifugation method possess few dead cells in their core region during the first $24-48 \mathrm{~h}$. Since the majority of our co- incubation studies were done $48 \mathrm{~h}$ following spheroid formation, they did not have the extensive necrotic core usually found in spheroids grown in the conventional manner, formed over a protracted time interval usually 1-2 weeks prior to their use.

Most biological tissue lack NIR-absorbing chromophores, thus permitting transmission of NIR light (700-1,000 nm). Therefore, normal tissue along the laser path should suffer minimal thermal damage, which is not the case for radiofrequency (RF) or magnetic thermal ablation [31, 32]. Spheroids containing empty Ma were not affected by even the highest laser irradiances tested $(28 \mathrm{~W} /$ $\mathrm{cm}^{2}$ ) Live/dead assays clearly demonstrated similar numbers of dead cells in spheroids containing empty $\mathrm{Ma}$ independent of laser treatment (Fig. 5a, b), in sharp contrast to the effects of NIR on spheroids containing loaded Ma where the overwhelming majority of tumor cells were dead (Fig. 5c, d).

The ability of PTT to only affect tumor cells adjacent to NS loaded Ma could be dramatically demonstrated in hybrid spheroids where the NS loaded Ma were constrained to the core region. Complete ablation of the core region was obtained (Fig. 8) while the surrounding tumor cells were still viable. Since the laser beam size was $3 \mathrm{~mm}$ and the spheroid had a diameter of $0.7 \mathrm{~mm}$, the entire spheroid received full laser exposure but only the core where the NS loaded Ma were located was ablated.

Comparable spheroid models have been employed in several of our previous studies on the effects of photodynamic therapy (PDT) on spheroid growth [19]. Identical PDT parameters on multiple replicate cultures often led to a survival dichotomy with a majority of cultures showing no survival but with a significant percentage of spheroids surviving treatment. This effect was clearly light fluence dependent and treatment often led to a growth delay but not to a growth cessation. PDT is a very oxygen dependent toxic modality and, like ionizing radiation, does not have a dramatic effect on hypoxic cells, which are found in the more central portions of the spheroids. This did not seem to be the case for PTT where growth delay was not observed and all replicate cultures completely ceased to grow and, in fact, contracted in size following treatment (Fig. 6A, B). Irreversible thermal damage to cells is not oxygen dependent but is caused by inactivation of vital enzymes, structural protein denaturation, and conformational changes induced in RNA and DNA that are so severe that the usual repair mechanisms are themselves inactivated. The resulting disruption of organelles, and especially cell membranes, is incompatible with survival $[33,34]$. In principle, PTT should be effective on both well oxygenated and hypoxic cancer cells, providing adequate heating occurs i.e. temperatures over $50^{\circ} \mathrm{C}$ for a sufficient time interval. In many of the laser exposed cultures, the spheroids 
containing loaded Ma were transformed to an amorphous mass indicating that the collagen in the extracellular matrix in the tissue had undergone hyalinization, a clear heat induced process.

Although NS can infiltrate into spheroids [35, 36], and spheroids incorporating NS could be produced by the centrifugation method, PTT following direct incorporation of NS was not explored since NS, like the ones investigated in this study, do not cross the intact BBB [18]. In contrast, macrophages readily traverse the patent BBB where residual glioma cells are found following surgical resection and therefore these immune cells have potential application as delivery vehicles in therapeutic applications. Nanoparticles tend to accumulate in tumors by virtue of the so-called enhanced permeability and retention effect due to poorly organized and fenestrated tumor vasculature combined with poor lymphatic drainage. Although nanoparticles can penetrate the leaky vasculature in tumors, the extracellular matrix of solid tumors presents a transport barrier that limits penetration, decreasing the efficacy of nanoparticle mediated therapies [36]. Cells on the other hand, can migrate through the extracellular matrix, especially inflammatory cells like Ma. Ma injected i.v will either track to the tumor site or be eliminated in the reticulo-endothelial system. Methods which would increase the ability of exogenous loaded macrophages to migrate to the BAT, making them available for postoperative therapeutic modalities, are key to the possible implementation of this technique, in future patient treatments, and are the subject of ongoing in vitro and animal studies [37].

Acknowledgments This work was supported by the Health Sciences System of the Nevada System of Higher Education through the Inter-institutional Biomedical Research Activities Fund (IBRAF). Henry Hirschberg is grateful for the support of the Norwegian Radiumhospital Research Foundation. Portions of this work were made possible through access to the Laser Microbeam and Medical Program (LAMMP) and the Chao Cancer Center Optical Biology Shared Resource at the University of California, Irvine. The nanoshells were a generous gift from Nanospectra Biosciences, Inc.

Open Access This article is distributed under the terms of the Creative Commons Attribution Noncommercial License which permits any noncommercial use, distribution, and reproduction in any medium, provided the original author(s) and source are credited.

\section{References}

1. Huber J, Egleton R, Davis T (2001) Molecular physiology and pathophysiology of tight junctions in the blood-brain barrier. Trends Neurosci 24:719-725

2. Ballabh P, Braun A, Nedergaard M (2004) The blood-brain barrier: an overview: structure, regulation, and clinical implications. Neurobiol Dis 16:1-13
3. Abbott N, Romero I (1996) Transporting therapeutics across the blood-brain barrier. Mol Med Today 2:106-113

4. Abbott N (2005) Physiology of the blood-brain barrier and its consequences for drug transport to the brain. Int Congr Ser 1277:3-18

5. Pardridge W, Pardridge WM (2007) Drug targeting to the brain. Pharm Res 24:1733-1744

6. Hickey WF (1999) Leukocyte traffic in the central nervous system: the participants and their roles. Semin Immunol 11:125-137

7. Badie B, Schartner JM (2000) Flow cytometric characterization of tumor associated macrophages in experimental gliomas. Neurosurgery 46:957-961 discussion 61-62

8. Roggendorf W, Strupp S, Paulus W (1996) Distribution and characterization of microglia/macrophages in human brain tumors. Acta Neuropathol 92:288-293

9. Strik HM, Stoll M, Meyermann R (2004) Immune cell infiltration of intrinsic and metastatic intracranial tumours. Anticancer Res 24:37-42

10. Shinonaga M, Chang CC, Suzuki N, Sato M, Kuwabara T (1988) Immunohistological evaluation of macrophage infiltrates in brain tumors Correlation with peritumoral edema. J Neurosurg 68:259-265

11. Valable S, Barbier EL, Bernaudin M, Roussel S, Segebarth C, Petit $\mathrm{E}$ et al (2008) In vivo MRI tracking of exogenous monocytes/macrophages targeting brain tumors in a rat model of glioma. Neuroimage 40(2):973-983

12. Wu J, xYang S, Luo H, Zeng L Lingbing Ye L, Lu Y. (2006) Quantitative evaluation of monocyte transmigration into the brain following chemical opening of the blood-brain barrier in mice. Brain Res 1098:79-85

13. Owen MR, Byrne HM, Lewis CE (2004) Mathematical modeling of the use of macrophages as vehicles for drug-delivery to hypoxic tumour sites. J Theor Biol 226:377-391

14. Choi MR, Stanton-Maxey KJ, Stanley JK, Levin CS, Bardhan R, Akin D et al (2007) A cellular Trojan Horse for delivery of therapeutic nanoparticles into tumors. Nano Lett 7(12): 3759-3765

15. Dou H, Destache CJ, Morehead JR et al (2006) Development of a macrophage-based nanoparticle platform for antiretroviral drug delivery. Blood 108:2827-2835

16. Hirsch LR, Gobin AM, Lowery AR, Tam F, Drezek RA, Halas $\mathrm{NJ}$ et al (2006) Metal nanoshells. Ann Biomed Eng 34(1):15-22

17. Huang X, Qian W, El-Sayed IH, El-Sayed MA (2007) The potential use of the enhanced nonlinear properties of gold nanospheres in photothermal cancer therapy. Lasers Surg Med 39(9):747-753

18. Schwartz JA, Shetty AM, Price RE, Stafford RJ, Wang JC, Uthamanthil RK et al (2009) Feasibility study of particle-assisted laser ablation of brain tumors in orthotopic canine model. Cancer Res 69(4):1659-1667

19. Madsen SJ, Sun CH, Tromberg BJ, Cristini V, DeMagalhaes N, Hirschberg H (2006) Multicell tumor spheroids in photodynamic therapy. Lasers Surg Med 38(5):555-564

20. Ivascu A, Kubbies M (2006) Rapid generation of single-tumor spheroids for high-throughput cell function and toxicity analysis. J Biomol Screen 11:922

21. Hirschberg H, Samset E, Hole PK, Lote K (2006) Impact of intraoperative MRI on the results of surgery for high grade gliomas. J Minim Invasive Neurosurg 48(2):77-84

22. Stummer W, Pichlmeier U, Meinel T, Wiestler OD, Zanella F, Reulen HJ (2006) Fluorescence-guided surgery with 5-aminolevulinic acid for resection of malignant glioma: a randomized controlled multicentre phase III trial. Lancet Oncol 7:392-401

23. Stummer W, Reulen HJ, Meinel T, Pichlmeier U, Schumacher W, Tonn JC et al (2008) Extent of resection and survival in glioblastoma multiforme: identification of and adjustment for bias. Neurosurgery 62:564-576 
24. Kemper E, Boogerd W, Thuis I, Beijnen J, Tellingen O (2004) Modulation of the blood-brain barrier in oncology: therapeutic opportunities for the treatment of brain tumors. Cancer Treat Rev 30:415-423

25. Siegal T, Zylber-Katz E (2002) Strategies for increasing drug delivery to the brain. Clin Pharmacokinet 41:171-186

26. Madsen SJ, Hirschberg H (2010) Site-specific opening of the blood-brain barrier. J Biophotonics 3(5-6):356-367

27. Metz S, Bonaterra G, Rudelius M, Settles M, Rummeny EJ, Daldrup-Link HE (2004) Capacity of human monocytes to phagocytose approved iron oxide MR contrast agents in vitro. Eur Radiol 14(10): 1851-1858

28. Engberink RD, Blezer EL, Hoff EI, van der Pol SM, van der Toorn A, Dijkhuizen RM et al (2008) MRI of monocyte infiltration in an animal model of neuroinflammation using SPIOlabeled monocytes or free USPIO. J Cereb Blood Flow Metab 28(4):841-851

29. Kah JC, Wong KY, Neoh KG, Song JH, Fu JW, Mhaisalkar S et al (2009) Critical parameters in the pegylation of gold nanoshells for biomedical applications: an in vitro macrophage study. J Drug Target 17(3):181-193

30. Storm G, Belliot SO, Daemen T, Lasic DD (1995) Surface modification of nanoparticles to oppose uptake by the mononuclear phagocyte system. Adv Drug Deliv Rev 17:31-48
31. Hirsch LR, Stafford RJ, Bankson JA et al (2003) Nanoshell mediated near-infrared thermal therapy of tumors under magnetic resonance guidance. Proc NatlAcad Sci USA 100:13549-13554

32. O'Neal DP, Hirsch LR, Halas NJ, Payne JD, West JL (2004) Photo-thermal tumor ablation in mice using near infraredabsorbing nanoparticles. Cancer Lett 209:171-176

33. Huang X, Jain PK, El-Sayed IH, El-Sayed MA (2006) Determination of the minimum temperature required for selective photothermal destruction of cancer cells with the use of immunotargeted gold nanoparticles. Photochem Photobiol 82(2):412417

34. Everts M (2007) Thermal scalpel to target cancer. Expert Rev Med Devices 4(2):131-136

35. Goodman TT, Olive PL, Pun SH (2007) Increased nanoparticle penetration in collagenase treated multicellular spheroids. Int $\mathbf{J}$ Nanomed 2(2):265-274

36. Goodman TT, Chen J, Matveev K, Pun SH (2008) Spatio-temporal modeling of nanoparticle delivery to multicellular tumor spheroids. Biotechnol Bioeng 101:388-399

37. Hirschberg H, Baek SK, Kwon YJ, Sun CH, Steen J, Madsen SJ (2010) Bypassing the blood brain barrier: delivery of therapeutic agents by macrophages. In: Proceedings SPIE-photonic therapeutics and diagnostics, vol 7548, 75483Z-1-5 